\title{
A Space Unrestricted Multi-Robot Combat Internet of Things System
}

\author{
Omar Said $^{1,2}$, Mehedi Masud ${ }^{1}$ \\ ${ }^{1}$ Computer Science Department, Taif University, Taif, KSA \\ ${ }^{2}$ College Science, Menoufia University, Shebin El Kom, Egypt \\ Email: o.saeed@tu.edu.sa,mmasud@scientist.com
}

Received May 9, 2012; revised June 16, 2012; accepted June 24, 2012

\begin{abstract}
Multi-robot cooperation problem has received increasing attention in the research community and has been extensively studied from different aspects. Space constrain problem is a major issue for building a multi-robot system. This constrain is a major hindrance for the efficient cooperation among robots in multi-robot applications. In this paper, we demonstrate a novel architecture of a multi-robot system without space restriction. Our architecture is based on the Internet of Things technology. We validated the proposed architecture using a case study considering a multi-robot combat application.
\end{abstract}

Keywords: Multi-Robot Systems; Internet of Things; RFID; Combat Applications

\section{Introduction}

Multi-robot cooperation systems play advanced role in the complex missions and applications. Multi-robot systems can accomplish many tasks where a single robot cannot accomplish. A group of robots accomplishes a target mission in a faster and reliable way compared to a single robot mission [1,2]. This is due to the distribution of tasks among the robots of a group. There are two types of cooperation between robots: 1) passive and 2) active. In the passive cooperation, the communication among robots is adhoc basis. In the active cooperation, the communication among robots is fixed and uses a short-distanced communication media. Generally, radios, wired electronic communication, and optical [3] medias which are used in the communication.

Multi-robot Systems improve the performance where the existing systems deploy single robot [4-7]. The cornerstone of a multi-robot system is the use of a wide range communication. If the system covers a small area, the benefits of using robots will be limited. Furthermore, the applicability of the multi-robot system in critical missions like terrorism and military becomes unusable.

Internet technology has become more prevalent in our lives in a shorter period of time than any other technology in the history. It has revolutionized in the way of people communicate with each other. Recently, the concept of the Internet as a set of connected computer devices is changed to a set of connected surrounding things of human's living space, such as home appliances, ma- chines, transportation, business storage, and goods etc. The number of things in the living space is larger than the number of world population. Research is going on how to make these things to communicate with each other like computer devices communicate through Internet. The communication among these things is referred as Internet of Things (IoT). Each element of IoT is called a thing and should have a unique address. Things communicate using the Radio-Frequency Identification (RFID) technology or the unique Internet Protocol address (IPV6). In addition, an intelligent web system is used to manage the communication. For more formalized definition of IoT can be found in $[8,9]$.

Considering the advent of IoT, this paper focuses on the wide area communication between robots using Internet of Things (IoT) technology (i.e. interaction via a communication link over wide distances). Particularly, we propose a multi-robot system based on the internet of things technology. The proposed multi-robot system can be used in many applications like health, space, and military. We present a case study that demonstrates how the anti-terrorism human team in a combat field can be transformed to the anti-terrorism multi-robot system.

The rest of the paper proceeds as follows. Section 2 demonstrates proposed multi-robot system architecture. Section 3 introduces the functionality of the multi-robot system. Section 4 introduces a multi-robot case study. Section 5 presents expected advantages of our proposed system. Finally, the paper concludes in Section 6. 


\section{Proposed Multi-Robot System Architecture}

Figure 1 shows the proposed multi-robot system which is based on the IoT technology. The proposed multi-robot architecture consists of four layers: 1) the robot layer, 2) the local IoT layer, 3) the translator layer, and 4) the global IoT layer. The robot layer defines the infrastructure of each robot and is mainly related to the robot's hardware. Hardware provides a robot's movements and abilities. The examples of hardware are power supply, motors, camera, microphone, sensor, driving mechanism, and etc. The local IoT layer contains the application that controls and executes a robot in a plane. The application is based on the Radio Frequency Identification (RFID) [10]. The translator layer is used for interoperability of messages between different robots' applications since the application of each robot may be designed using different languages. The fourth layer contains a global application which is used to control the multi-robot system. The global application is an intelligent web application.

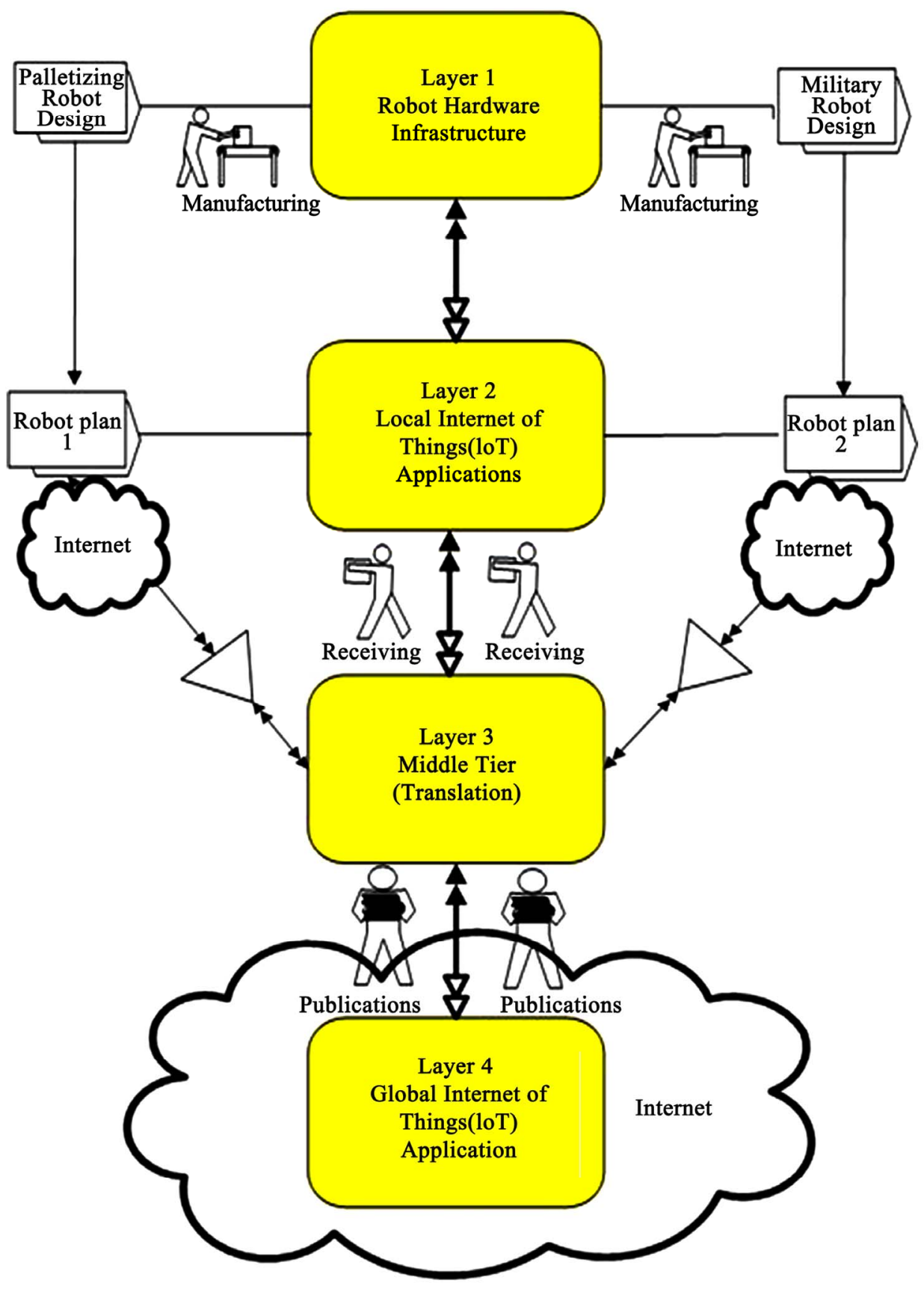

Figure 1. Our proposed system architecture. 


\section{Functionality of Multi-Robot System}

In a multi-robot system, a robot should be able to accomplish a specified mission when it is sent for a mission task. In order to execute a task the robot application in a robot needs data from the environment to make a suitable step. Feeding each robot application with environmental data is a major factor that affects the robot's action accuracy. There are three techniques to provide data to a robot from the environment. In the first technique, data is provided from a plane that is used to control and accomplish the robot mission. In the second technique, data is provided by human control techniques. In the third technique, data is provided to the robot using cameras or sensors, which collect multimedia information from the robot's environment. Sometime a robot needs information which is not available in the surrounding environment or is covered by sensors or cameras. In the proposed system each robot acts like a human. A robot provides other robots with data, which may be required to run live actions. In the multi-robot system, there is no human intervention. To eliminate the human intervention, an intelligent communication system [11-13], which simulates a human behavior between robots, is used. Scalable and wide range communication challenge is solved using the Internet as a communication media. The Internet is widely used technology; hence it can be considered as an ideal media to transmit instructions and environmental information which is collected by different robots settled in different positions. If the second layer needs a data for a robot to accomplish its mission, it fires a request to the fourth layer. The fourth layer then distributes the robot request on the other robot systems. The data is sent to the robot using the Internet. The third layer is used to translate the robot requests received from the second layer in case of using different languages.

In the system, each robot is equipped with the DualBand UHF-RFID Tags [14] to collect data and distribute it through a wireless communication media. Each robot sends and receives data (instructions or environment information) by a dual communication web application. Each robot is addressed using IPV6 address space which is discussed in the IoT technology [15].

\section{Multi-Robot Case Study}

For the case study, we consider a combat mission against terrorism which is generally accomplished by human team. We show how this mission can be performed using the proposed multi-robot system. The case study shows that the proposed system provides better performance. Furthermore, it demonstrates a long run solution for some critical missions, such as combating terrorism and war. This case study is difficult (if not impossible) to be accomplished by the current multi-robot systems, which miss many characteristics such as autonomously, scalability, and wide range communication.

In the proposed multi-robot system, we replace each human in the mission by a robot. Each solder target is executed by a robot. In the system, human's sense capability is achieved by a robot using sensors, the vision capability is achieved by cameras, the speaking capability is achieved by data transmission between robots, the thinking capability is achieved by artificial intelligence techniques [16], and the prediction capability is solved by feedback algorithms [17]. Thinking and prediction capability of each robot depends on the data that are collected by the sensors and cameras. In a combat mission, the error percentage in the actions should be near to zero. So, the collected data is a very important factor in robot's reactions. The collected data depends on the number of robots and the range of communication which is covered by the robots, the data collection time, and the environment from which the data are collected.

In Figure 2(a) we show the scenario of executing the mission using human combat team. The target of the mission is to fight a terrorist and capture as live or dead. The combat team consists of four well trained soldiers. These soldiers are equipped with wireless communication tools and guns.

In Figure 2(b) we show how the mission in Figure 2(a) is transformed into a multi-robot system. Each solider is replaced by a fully equipped robot (camera, sensors, battery, etc.). Each robot has RFID tag and a wireless card for Internet connection. The warrior tools can be attached on the robot. Each robot is able to walk or move on a rocky land. Each robot in the mission is fixed with a target and can cooperate with other robots. Let us imagine a state to explain how a robot in the multi-robot system cooperates with other robots. Suppose that a terrorist moves from on region to another. One of the robots which are responsible about this region observation sends an alarm to other robots informing the new state (position, style, and equipments) of the terrorist. Hence, a robot form the team can shoot the terrorist. The shooting moment is determined depending on the thinking and the prediction.

\section{Expected Advantages of Our Proposed System}

Our system has following advantages.

Scalability: the proposed system can support a huge number of robots due to natural of the connection media (Internet). The system has an ability to enhance the system by adding new functionality to the local or global web intelligent applications. Furthermore, there is enormous amount of information available through the Internet. This information may be used in the multi-robot 
system during the mission execution. For example the Google Earth Internet application may be used to describe the target environment which may be real time changed by some outside factors such as atmosphere.

Minimal additional hardware complexity: no notable additional hardware is needed to accomplish the communication between the robots. The only attached hardware is the RFID tag or reader.

Multi applications suitability: our system opens the door to collect a single robot's applications to be one strong application used in big missions.

Wide distance communication: nowadays, the Internet technology is supported by satellite, which means that the media of robot communication is available anywhere.

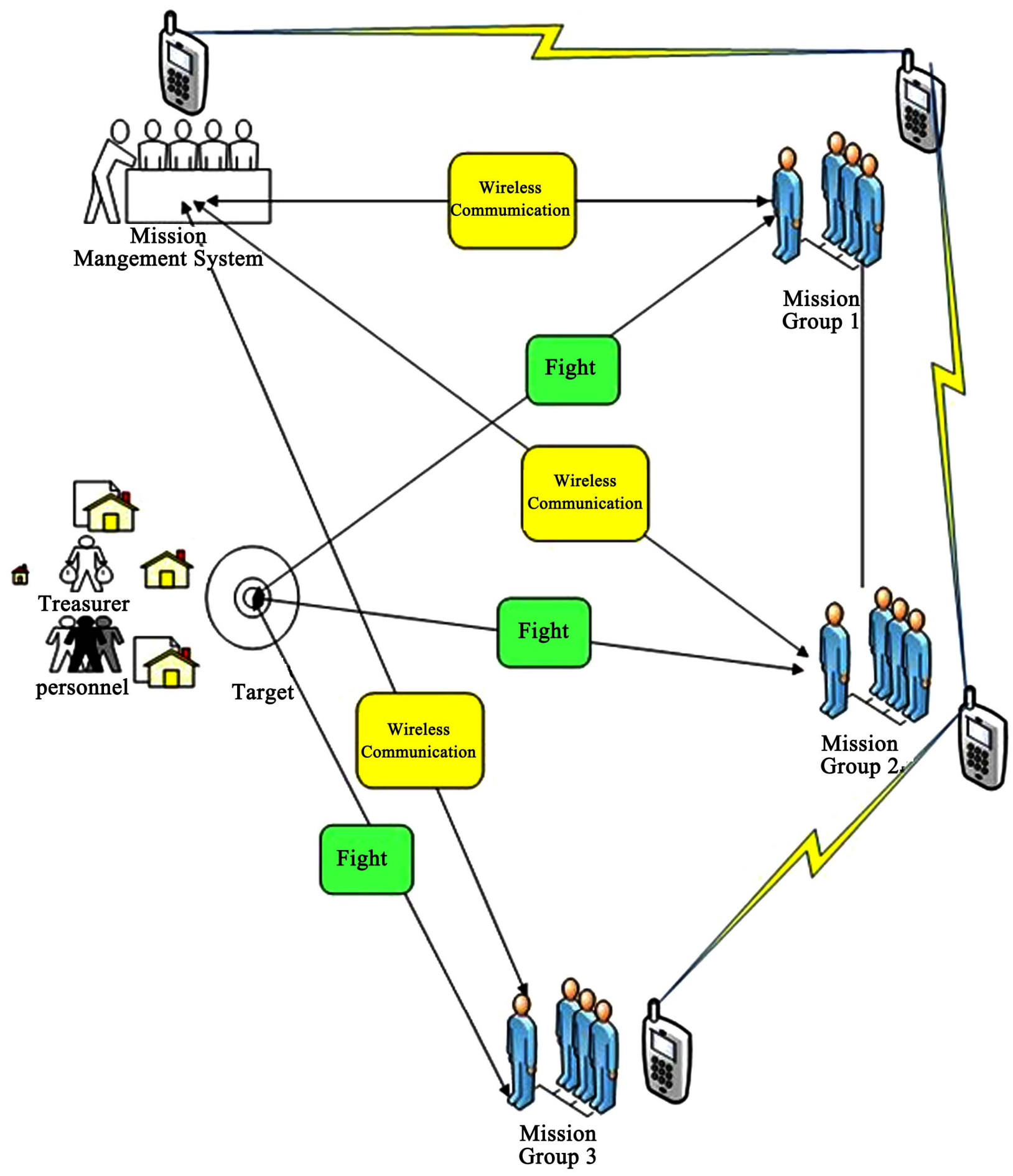

(a) 


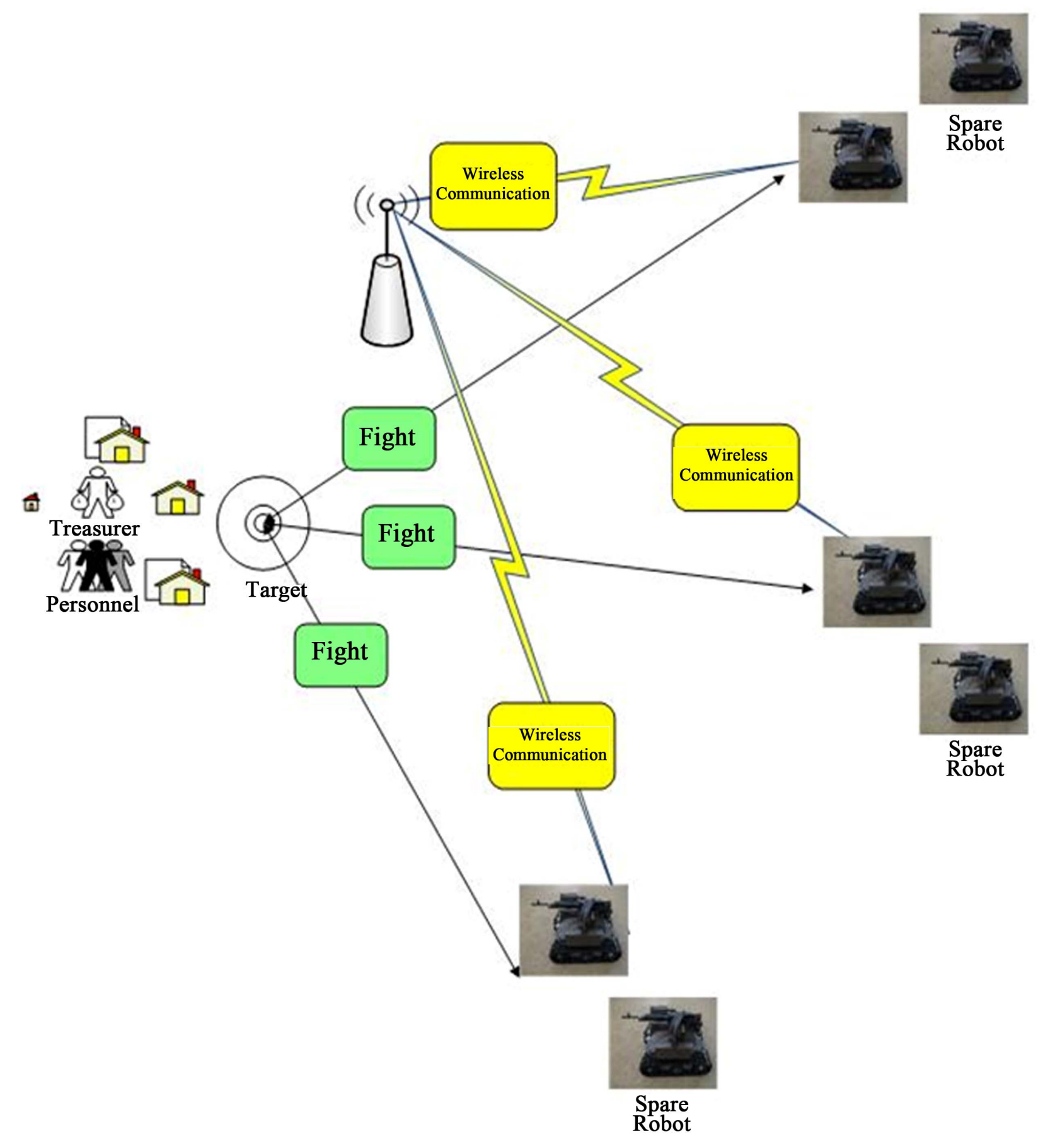

(b)

Figure 2. Human (a) vs Robots (b) in terrorism mission case study.

Mission distribution: the mission is distributed in our multi-robot systems. This makes the execution of each mission part precious and fast. In addition, when the number of robots increases, each mission part will be simple, which means that the efficiency of part excursion increases. The mission distribution means not only each robot should have one part; but also, it may help in other mission parts for other robots team. In addition, sharing information between robots provide each robot with ambiguous data which may be used in its mission part.

No human intervention: this is a very important issue because in some cases like terrorism or military missions, the human sharing may lead to death or arrest. So, when we exchange the human with robots, the loss percentage 
in human and information will be eliminated.

\section{Conclusion}

In this paper, multi-robot system architecture is described based on the internet of things technology. The IoT provides the multi-robot system with scalability, wide range recovering, and easy communication. The system architecture contains 4 layers, one hardware layer and 3 software layers. The multi-robot system can be used in many applications like health, space, and military. Terrorism case study for our multi-robot system is demonstrated to clarify how the anti-terrorism human team can be transformed to the anti-terrorism robots team. Finally, the advantages of the proposed system are discussed.

\section{Future Work}

In the proposed system, we have more challenges like the global IoT application construction (general plan of the proposed multi-robot system). Furthermore, the middle tier construction can be considered as a challenge due to different local robot applications and plans. Also, the security system is extremely important issue especially when we apply our proposed system in critical environment such as military or terrorism $[18,19]$. Other issues like scalability, accuracy, and reliability should be considered. In addition, a control system for army of robots, which contains three main groups; diver robots, fly robots, and ground robots, will be constructed (work in progress). Also, the hiding robot's ability issue will be scoped.

The paper mainly describes the foundation of developing a multi-robot system using IoT technology. We are currently working on the designing phase of the implementation part. Our future goal is also to implement the system in a real scenario.

\section{REFERENCES}

[1] D. Kato, K. Sekiyama and T. Fukuda, "Autonomous Cooperation Planning for Heterogeneous Multi-Robot," IEEE Workshop on Robotic Intelligence in Informationally Structured Space (RIISS), Nagoya, 11-15 April 2011, pp. 6368.

[2] Y. F. Cai and S. X. Yang, "Fuzzy Logic-Based MultiRobot Cooperation for Object-Pushing," International Conference on Information and Automation, Shenzhen, 6-8 June 2011, pp. 273-278.

[3] G. Dudek, M. Jenkin and E. Milios, "A Taxonomy of Multi-Robot Systems,” In: T. Balch and L. E. Parker, Eds., Robot Teams: From Diversity to Polymorphism, 2002, pp. 3-22.

[4] C. Y. Wong, G. Seet and S. K. Sim, "Multiple-Robot Systems for USAR: Key Design Attributes and Deployment Issues," International Journal of Advanced Robotic Systems, Vol. 8, No. 1, 2011, pp. 85-101.
[5] L. E. Parker, “Multiple Mobile Robot Systems,” In: B. Siciliano and O. Khatib, Eds., Springer Handbook of Robotics, Springer, Berlin, 2008, pp. 921-941. doi:10.1007/978-3-540-30301-5_41

[6] N. Shiroma, Y. H. Chiu, N. Sato and F. Matsuno, "Cooperative Task Execution of a Search and Rescue Mission by a Multi-Robot Team," Advanced Robotics, Vol. 19, No. 3, 2005, pp. 311-329. doi:10.1163/1568553053583670

[7] I. R. Nourbakhsh, K. Sycara, M. Koes, M. Yong, M. Lewis and S. Burion, "Human-Robot Teaming for Search and Rescue,” IEEE Pervasive Computing, Vol. 4, No. 1, 2005, pp. 72-77. doi:10.1109/MPRV.2005.13

[8] A. Luigi, I. Antonio and M. Giacomo, "The Internet of Things: A Survey," Science Direct Journal of Computer Networks, Vol. 54, No. 15, 2010, pp. 2787-2805.

[9] R. Odrigo and A. Cristina, "Key Management Systems for Sensor Networks in the Context of the Internet of Things," Computers and Electrical Engineering, Vol. 37, No. 2, 2011, pp. 147-159. doi:10.1016/j.compeleceng.2011.01.009

[10] Y. Chen, "Understanding Technology Adoption through System Dynamics Approach: A Case Study of RFID Technology," 9th International Conference on Embedded and Ubiquitous Computing (EUC), Melbourne, 24-26 October 2011, pp. 366-371. doi:10.1109/EUC.2011.75

[11] D. Portugal and R. Rocha, "A Survey on Multi-Robot Patrolling Algorithms,” IFIP International Federation for Information Processing, Vol. 349, 2011, pp. 139-146.

[12] M. L. Wang, Z. C. Jia and C. G. Yang, "A New Framework of Optimal Multi-Robot Formation Problem,” 30th Chinese Control Conference, Yantai, 22-24 July 2011, pp. 4139-4144.

[13] J. Leitner, "Multi-Robot Formations for Area Coverage in Space Applications,” Teknillinen Korkeakoulu Faculty of Electronics, Communications and Automation Department of Automation and Systems Technology, 2011.

[14] F. Paredes, et al., "Dual-Band UHF-RFID Tags Based on Meander-Line Antennas Loaded with Spiral Resonators," IEEE Antennas and Wireless Propagation Letters, Vol. 10, 2011, pp. 768-771. doi:10.1109/LAWP.2011.2162716

[15] Y.-W. Ma, C.-F. Lai, Y.-M. Huang and J.-L. Chen, "Mobile RFID with IPv6 for Phone Services," Proceedings of IEEE ISCE, Kyoto, 25-28 May 2009, pp. 169-170.

[16] B. Johnen, C. Scheele and B. Kuhlenkotter, "Learning Robot Behavior with Artificial Neural Networks and a Coordinate Measuring Machine,” International Conference on Automation, Robotics and Applications (ICARA), Dortmund, 6-8 December 2011, pp. 208-213.

[17] D. T. Liu, Y. Peng and X. Y. Peng, "Online Adaptive Status Prediction Strategy for Data-Driven Fault Prognostics of Complex Systems," International Conference on Prognostics and System Health Management, Shenzhen, 12-15 September 2011, pp. 1-6. doi:10.1109/ICPHM.2011.6024364

[18] L. Zhou and H.-C. Chao, "Multimedia Traffic Security Architecture for the Internet of Things," IEEE Networks, Vol. 25, No. 3, 2011, pp. 35-40. doi:10.1109/MNET.2011.5772059 
[19] X. Li, X. Zhou and W. Liu, "Research on the Architecture of Trusted Security System Based on the Internet of Things,” Fourth International Conference on Intelligent
Computation Technology and Automation, Shanghai, 28-29 March 2011, pp. 1172-1175.

doi:10.1109/ICICTA.2011.578 